\title{
Time-Delayed Impulsive Control of Chaotic System Based on T-S Fuzzy Model
}

\author{
Cheng Hu and Haijun Jiang \\ College of Mathematics and System Sciences, Xinjiang University, Urumqi, Xinjiang 830046, China \\ Correspondence should be addressed to Cheng Hu; hucheng@xju.edu.cn
}

Received 19 May 2014; Revised 5 August 2014; Accepted 7 August 2014; Published 27 August 2014

Academic Editor: Hak-Keung Lam

Copyright (C) 2014 C. Hu and H. Jiang. This is an open access article distributed under the Creative Commons Attribution License, which permits unrestricted use, distribution, and reproduction in any medium, provided the original work is properly cited.

\begin{abstract}
This paper is concerned with the time-delayed impulsive control and synchronization of general chaotic system based on T-S fuzzy model. By utilizing impulsive control theory, time-delayed feedback control technique, and T-S fuzzy model, some useful and new conditions are derived to guarantee the stability and synchronization of the addressed chaotic system. Finally, some numerical simulations are given to illustrate the effectiveness of the derived results.
\end{abstract}

\section{Introduction}

Chaos may well be considered together with relativity and quantum mechanics as one of the three monumental discoveries of the twentieth century [1]. The term chaos associated with an interval map was first formally introduced into mathematics by Li and Yorke in 1975, where they established a simple criterion for chaos in one-dimensional difference equations, that is, the well-known "period three implies chaos." Initially regarded as a curiosity that interested only the mathematics community, it was later revamped when observed in meteorology, physics, chemistry, biology, and so on [2]. Over the past four decades, chaos has matured as a science and has given us deep insights into previously intractable and inherently nonlinear natural phenomena. There is, however, still no unified, universally accepted, and rigorous mathematical definition of chaos in the scientific literature to provide a fundamental basis for studying such exotic phenomena. Roughly speaking, chaotic behavior is a seemingly random behavior of a deterministic system that is characterized by sensitive dependence on initial conditions. From a practical point of view, a chaotic motion can be defined as a bounded invariant motion of a deterministic system, that is, not an equilibrium solution or a periodic solution or a quasiperiodic solution [2].
Although chaos is a very attractive subject for study, it was once believed to be neither predictable nor controllable due to its intrinsic topological complexity. However, recent research has shown that chaos can actually be useful under certain circumstances, such as enhanced mixing of chemical reactants. On the other hand, chaos should be weakened or completely suppressed when it is harmful. Therefore, it is very necessary and important to investigate chaos and chaos control.

Currently, many different techniques and methods based on conventional control theory have been extensively investigated to achieve chaos control [3-11]. For instance, the socalled OGY control method, introduced by Ott et al. [7], employs the classical feedback control idea, which might be understood as a kind of pole-placement method and is technically quite simple, but virtually it takes advantage of chaos itself in the sense of using its structural stability and its basic property of having a dense set of periodic orbits near a saddle. Such special properties are not available for nonchaotic systems. Therefore, such a control methodology was not first recommended by control theorists and engineers who usually, if not always, try to use brute force to regulate and stabilize unstable dynamics [1]. When brute force type of controls is not allowed, for example, in fragile and microscopic biological control systems such 
as human brain and heart regulations, new control methods utilizing the extreme sensitivity of chaos to tiny variations are very desirable. This usually leads to some nonconventional approaches.

Fuzzy control as a nontraditional method of control, in recent years, has received much attention as a powerful tool for the nonlinear control. Compared with the traditional control paradigm, the advantages of the fuzzy control paradigm are twofold. Firstly, a precise mathematical model of the controlled system is not required. In addition, a satisfactory nonlinear controller can often be developed empirically without using complicated mathematics [1]. In view of its superiority, fuzzy controllers are often used for controlling conventional control methods as an extra layer of control in order to improve the effectiveness of the control.

On the other hand, the main drawback of fuzzy control systems is the lack of a systematic modeling and control design methodology. Particularly, stability analysis of fuzzy system is not easy, and parameter tuning is generally a timeconsuming procedure, due to the nonlinear and multiparametric nature of fuzzy control systems [2]. To resolve these problems, a linear system can be adopted as the consequent part of a fuzzy rule, which leads to the so-called T-S model.

The T-S fuzzy dynamic model originates from Takagi and Sugeno. In this framework, a nonlinear dynamical system is first approximated by a T-S fuzzy model. In this type of fuzzy model, local dynamics in different state-space regions are represented by linear models. The overall model of the system is achieved by fuzzy blending of these linear models. The control design is carried out based on the fuzzy model. For each local linear model, a feedback control is designed. The resulting overall controller, which is nonlinear in general, is again a fuzzy blending of each individual controller $[1,2]$. Here, it is noted that the defuzzified output of the constructed T-S fuzzy model is mathematically identical to that of the original nonlinear system. Clearly, the T-S fuzzy model provides a successful method to describe certain complex nonlinear systems using some local linear subsystems. In view of its convenience, T-S fuzzy control theory has been extensively applied to chaos control and synchronization in some literatures [12-21].

In addition, impulsive control method has been also widely used to stabilize and synchronize chaotic systems in recent years [13, 16, 18-27]. Impulsive control gives an efficient method to deal with systems which cannot endure continuous disturbance. Additionally, in synchronization process, the driven system receives the information about the active variables of the driving system only in the discrete times. This drastically reduces the amount of information transmitted from the driving system to driven system which makes this method more efficient and more useful in a great number of applications, such as in encrypt communications; this point is essential [18].

The delayed feedback control, as one of the important chaos control means, was introduced by Lithuanian physicist K. Pyragas in 1992 to stabilize an unstable periodic orbit of a nonlinear control system $[1,2,9]$. Based on this method, the control input is fed by the difference between the current state and the delayed state. Currently, the delayed feedback control method has been extensively applied to controlling chaos $[8,9,12,22,28-34]$.

Although impulsive control, fuzzy control, and delayed feedback control are widely proposed in study of chaos and chaos control, to the best of our knowledge, there are few results on the stabilization and synchronization of chaotic system based on T-S fuzzy model by using the so-called time-delayed impulsive control which combine the superiorities of impulsive control and time-delayed feedback control. In addition, most previous researches have been restricted to linear impulsive control functions (e.g., see [18-20, 23, 25, 27]); few authors have considered the nonlinear impulsive control functions. Besides, the impulsive controllers are independent of time delays in most existing researches (see $[13,16,18-21,23-27]$ ). Motivated by the above discussion, the objective of this paper is to develop a time-delayed impulsive controller for stabilization and synchronization of general chaotic systems based on a $\mathrm{T}$ $S$ fuzzy model. In this paper, the impulsive controller can be a nonlinear function. In addition, because of using timedelayed feedback control technique, the delayed impulsive controllers are derived from controlling chaotic system; this extends some previous impulsive control results to some extent.

The paper is organized as follows. In Section 2, the general T-S fuzzy control model of chaotic system is presented by applying T-S fuzzy theory. In Section 3, by combining the time-delayed feedback control technique and the impulsive control method, some sufficient conditions are obtained to guarantee that the origin of the controlled chaotic system is globally asymptotically stable. In Section 4, some timedelayed impulsive synchronization conditions are provided. In Section 5, the effectiveness and feasibility of the developed methods have been shown by some numerical simulations.

\section{The T-S Fuzzy Control Model of Chaotic System}

A T-S fuzzy model is described by a set of if-then rules, which characterize local relations of a nonlinear system in the state space. The main feature of a T-S model is to express the local dynamics of each fuzzy rule by a linear state-space system model, and the overall fuzzy system is modeled by fuzzy "blending" of these local linear system models through some suitable membership functions.

Consider the following chaotic system:

$$
\dot{x}(t)=f(t, x(t)),
$$

where $x \in R^{n}$ is the state variable and $f \in C\left[R^{+} \times R^{n}, R^{n}\right]$ is a nonlinear vector-valued function satisfying $f(t, 0) \equiv 0$ for $t \in R^{+}$. We will construct a T-S fuzzy control model for (1) in the following form (see $[1,2])$.

$$
\begin{aligned}
& \text { Rule } i: \text { if } z_{1}(t) \text { is } M_{i}^{1}, z_{2}(t) \text { is } M_{i}^{2}, \ldots, z_{p}(t) \text { is } M_{i}^{p} \text {, then } \\
& \dot{x}(t)=A_{i} x(t)+u_{i}(t), \quad i=1,2, \ldots, r,
\end{aligned}
$$

where the premise variables $z_{1}(t), \ldots, z_{p}(t)$ are proper state variables; each $M_{i}^{j}$ and $u_{i}(t)$ denote the fuzzy set and the input 
control signal, respectively; each $A_{i}$ denotes a $n \times n$ constant matrix; $r$ is the number of the fuzzy rules.

Using the singleton fuzzifier, product fuzzy inference, and weighted average defuzzifier (see $[1,2]$ ), the final output of the T-S fuzzy control system (2) for system (1) is inferred as follows:

$$
\dot{x}(t)=\sum_{i=1}^{r} h_{i}(z(t))\left[A_{i} x(t)+u_{i}(t)\right],
$$

where $z(t)=\left(z_{1}(t), \ldots, z_{p}(t)\right)$ and

$$
h_{i}(z(t))=\frac{w_{i}(z(t))}{\sum_{i=1}^{r} w_{i}(z(t))}, \quad w_{i}(z(t))=\prod_{j=1}^{p} M_{i}^{j}\left(z_{j}(t)\right),
$$

in which $M_{i}^{j}\left(z_{j}(t)\right)$ is the grade of membership of $z_{j}(t)$ in $M_{i}^{j}$, $w_{i}(z(t)) \geq 0(i=1,2, \ldots, r)$, and $\sum_{i=1}^{r} w_{i}(z(t))>0$.

It is clear that

$$
\sum_{i=1}^{r} h_{i}(z(t))=1, \quad h_{i}(z(t)) \geq 0, i=1,2, \ldots, r
$$

for all $t$, where $h_{i}(z(t))$ can be regarded as the normalized weight of the if-then rules.

Remark 1. If the input control signal in system (2) is invalid, that is, $u_{i}(t) \equiv 0$ for any $t \in R^{+}$and $i=1,2, \ldots, r$, then system (1) can be represented by the following T-S fuzzy model.

Rule $i$ : if $z_{1}(t)$ is $M_{i}^{1}, z_{2}(t)$ is $M_{i}^{2}, \ldots, z_{p}(t)$ is $M_{i}^{p}$, then

$$
\dot{x}(t)=A_{i} x(t), \quad i=1,2, \ldots, r .
$$

Similar to the above discussion, the overall output of system (6) is inferred as follows:

$$
\dot{x}(t)=\sum_{i=1}^{r} h_{i}(z(t)) A_{i} x(t),
$$

where $z_{i}(t), M_{i}^{j}, A_{i}$, and $h_{i}(z(t))$ are defined in (2) and (3), respectively. In this case, system (1) is mathematically identical to the defuzzified output of the constructed T-S fuzzy model (7).

Finally, for convenience and further study, we introduce the following lemmas.

Lemma 2 (see [29]). For any two real column vectors $\zeta$, $\eta$ with the same dimensions and any positive constant $\theta$, the following inequality holds:

$$
2 \zeta^{T} \eta \leq \frac{1}{\theta} \zeta^{T} \zeta+\theta \eta^{T} \eta
$$

Lemma 3 (see [29]). Assume that $P$ is a $n \times n$ real symmetric matrix, and $\lambda_{1}$ and $\lambda_{2}$ are the smallest and the largest eigenvalues of $P$, respectively. Then for any $\xi \in R^{n}$, the following inequality holds:

$$
\lambda_{1} \xi^{T} \xi \leq \xi^{T} P \xi \leq \lambda_{2} \xi^{T} \xi .
$$

\section{Time-Delayed Fuzzy Impulsive Control}

In this section, we will design a time-delayed impulsive controller for chaotic system (1) based on fuzzy model (2). In the following, we assume that the controller shares the same fuzzy sets with system (2). More precisely, the $i$ th control rule is formulated in the following form.

Control rule $i$ : if $z_{1}(t)$ is $M_{i}^{1}, z_{2}(t)$ is $M_{i}^{2}, \ldots, z_{p}(t)$ is $M_{i}^{p}$, then

$$
u_{i}(t)=\sum_{k=1}^{\infty} F_{i} l_{k}(t) x(t)+\sum_{k=1}^{\infty} \delta(t-k T) I_{i k}(x(t), x(t-T))
$$

for $i=1,2, \ldots, r$, where $T>0$ is the feedback time-delay, each $F_{i}$ denotes $n \times n$ constant matrix, the switching function $l_{k}(\cdot)$ is defined by the following form:

$$
l_{k}(t)=\left\{\begin{array}{lc}
1, & (k-1) T<t \leq k T \\
0, & \text { otherwise }
\end{array}\right.
$$

$\delta(\cdot)$ is the Dirac delta function, each $I_{i k}(\cdot) \in C\left[R^{n} \times R^{n}, R^{n}\right]$ denotes the additive change of the state at time $k T$ satisfying $I_{i k}(0,0)=0$, and the following condition is also satisfied.

$(H)$ There exist constants $a_{i k}>0$ and $b_{i k} \geq 0$ such that

$$
\left\|u+I_{i k}(u, v)\right\|^{2} \leq a_{i k}\|u\|^{2}+b_{i k}\|v\|^{2}
$$

for any $u, v \in R^{n}$. Here and throughout this paper, $\|y(t)\|=$ $\left[y^{T}(t) y(t)\right]^{1 / 2}$ is the Euclidean norm.

From (10), system (3) can be rewritten as follows:

$$
\begin{aligned}
\dot{x}(t)= & \sum_{i=1}^{r} h_{i}(z(t))\left[A_{i} x(t)+u_{i}(t)\right] \\
= & \sum_{i=1}^{r} h_{i}(z(t)) A_{i} x(t) \\
& +\sum_{i=1}^{r} h_{i}(z(t)) \sum_{k=1}^{\infty} F_{i} l_{k}(t) x(t) \\
& +\sum_{i=1}^{r} h_{i}(z(t)) \sum_{k=1}^{\infty} \delta(t-k T) I_{i k}(x(t), x(t-T)),
\end{aligned}
$$

which implies that

$$
\begin{aligned}
& x(k T+h)-x(k T) \\
&=\int_{k T}^{k T+h}\left\{\sum_{i=1}^{r} h_{i}(z(t)) A_{i} x(t)\right. \\
& \\
& \quad+\sum_{i=1}^{r} h_{i}(z(t)) \sum_{k=1}^{\infty} F_{i} l_{k}(t) x(t)
\end{aligned}
$$




$$
\begin{aligned}
& +\sum_{i=1}^{r} h_{i}(z(t)) \\
& \left.\times \sum_{k=1}^{\infty} \delta(t-k T) I_{i k}(x(t), x(t-T))\right\} d t,
\end{aligned}
$$

where $h>0$ is sufficiently small. As $h \rightarrow 0^{+}$, by applying the properties of the Dirac delta function, we obtain

$$
x\left(k T^{+}\right)-x(k T)=\sum_{i=1}^{r} h_{i}(z(k T)) I_{i k}(x(k T), x((k-1) T)) .
$$

In this paper, we assume that $x(t)=\left(x_{1}(t), x_{2}(t), \ldots, x_{n}(t)\right)^{T}$ is left continuous at $k T\left(k \in Z^{+}\right)$; that is, $x_{i}(k T)=$ $\lim _{t \rightarrow k T^{-}} x_{i}(t)$. Thus, system (13) is rewritten as

$$
\begin{aligned}
\dot{x}(t) & =\sum_{i=1}^{r} h_{i}(z(t))\left(A_{i}+F_{i}\right) x(t), \quad t \neq k T, \\
\Delta x(t) & =\sum_{i=1}^{r} h_{i}(z(t)) I_{i k}(x(t), x(t-T)), \quad t=k T, k \in Z^{+} .
\end{aligned}
$$

Remark 4. Evidently, the control input $u_{i}(t)$ is an $n$-dimensional vector function denoted by $\left(u_{i 1}(t), u_{i 2}(t), \ldots, u_{i n}(t)\right)^{T}$ and it will converge to zero if the origin is asymptotically stable. On the other hand, if controller (10) is invalid, that is, $u_{i}(t) \equiv 0$ for all $t \geq 0$, then it is easy to see that system (16) is equivalent to system (7) in this case. In addition, it is evident that $x(t)=0$ is an equilibrium of system (16).

In the following, we discuss the globally asymptotical stability of the origin of system (16). Firstly, for convenience, we introduce the following mathematical denotations.

Let $P$ be a symmetric and positive definite matrix and $\lambda_{1}>0$ and $\lambda_{2}>0$ are the smallest and the largest eigenvalues of $P$, respectively.

$\beta_{i}$ denotes the largest eigenvalue of $P\left(A_{i}+F_{i}\right)+\left(A_{i}+F_{i}\right)^{T} P$. $\alpha=\max _{1 \leq i \leq r}\left\{\beta_{i}\right\}, \beta=\alpha / \lambda_{1}$, and

$$
\begin{array}{ll}
H_{k}=\frac{\left(\theta^{2}+1\right) \lambda_{2}}{2 \theta \lambda_{1}} \sum_{i=1}^{r} h_{i}(z(k T)) a_{i k}, & k \in Z^{+}, \\
B_{k}=\frac{\left(\theta^{2}+1\right) \lambda_{2}}{2 \theta \lambda_{1}} \sum_{i=1}^{r} h_{i}(z(k T)) b_{i k}, \quad k \in Z^{+},
\end{array}
$$

where $\theta>0$ is an arbitrary real number.

$G(t): R^{+} \rightarrow R^{+}$denotes a differentiable at $t \neq k T$ and bounded function; that is, there exist $m>0$ and $M>0$ such that

$$
m \leq G(t) \leq M, \quad \forall t \in R^{+}
$$

Furthermore, denote $\eta=M \lambda_{2} / m \lambda_{1}$ and

$$
L_{k}=H_{k}+\frac{B_{k}}{\eta L_{k-1}} \exp (-\beta T) \frac{G\left((k-1) T^{+}\right)}{G(k T)}
$$

for $k \in Z^{+}$, where $L_{0}=1$.

Remark 5. From $a_{i k}>0$ in $(H)$, we know that $H_{k}>0$ for $k \in Z^{+}$, which implies that the definition of $L_{k}$ is reasonable.

The following theorem is provided to guarantee that the origin of system (16) is asymptotically stable.

Theorem 6. Under assumption $(H)$, if there exists a constant $\theta>0$ such that one of the following conditions is satisfied:

(1) $\beta \geq 0$ and there exists a constant $\mu>1$ such that

$\beta T+\ln \left(\mu \eta L_{k}\right)+\ln \frac{G((k+1) T)}{G\left(k T^{+}\right)} \leq 0, \quad \forall k \in Z^{+}$,

(2) $\beta<0$ and there exists a constant $\varepsilon$ satisfying $0 \leq \varepsilon<$ $-\beta$ such that

$$
\ln \left(\eta L_{k}\right)-\varepsilon T+\ln \frac{G((k+1) T)}{G\left(k T^{+}\right)} \leq 0, \quad \forall k \in Z^{+},
$$

then the controlled system (16) is globally asymptotically stable at its origin.

Proof. Choose a Lyapunov function as follows:

$$
V(x(t))=x^{T}(t) P x(t) .
$$

Let $W(t)=G(t) V(x(t))$. For $t \neq k T$, the upper right derivative of $W(t)$ along the solution of system (16) is

$$
\begin{aligned}
& D^{+} W(t) \\
& =G(t) D^{+} V(x(t))+D^{+} G(t) V(x(t)) \\
& =G(t) \sum_{i=1}^{r} h_{i}(z(t)) x^{T}(t) \\
& \quad \times\left[\left(A_{i}+F_{i}\right)^{T} P+P\left(A_{i}+F_{i}\right)\right] \\
& \quad \times x(t)+\dot{G}(t) V(x(t)) \\
& \leq G(t) \sum_{i=1}^{r} h_{i}(z(t)) \beta_{i} x^{T}(t) x(t)+\dot{G}(t) V(x(t)) \\
& \leq\left(\beta+\frac{\dot{G}(t)}{G(t)}\right) G(t) V(x(t)) \\
& =\left(\beta+\frac{\dot{G}(t)}{G(t)}\right) W(t) .
\end{aligned}
$$

Let $p(t)=\beta+(\dot{G}(t) / G(t))$; then we have

$D^{+} W(t) \leq p(t) W(t), \quad t \in((k-1) T, k T], k \in Z^{+}$, 
which implies that

$$
W(t) \leq W\left((k-1) T^{+}\right) \exp \left[\int_{(k-1) T^{+}}^{t} p(s) d s\right] .
$$

It follows that

$$
\omega(t) \leq \eta \omega\left((k-1) T^{+}\right) \exp \left[\int_{(k-1) T^{+}}^{t} p(s) d s\right]
$$

for $t \in((k-1) T, k T]$ and $k \in Z^{+}$, where $\omega(t)=x^{T}(t) x(t)$ and $\eta=M \lambda_{2} / m \lambda_{1} \geq 1$.

For $t=k T\left(k \in Z^{+}\right)$, we obtain

$$
\begin{aligned}
& V\left(x\left(k T^{+}\right)\right) \\
& =V(x(k T)+\Delta x(k T)) \\
& =[x(k T) \\
& \left.\quad+\sum_{i=1}^{r} h_{i}(z(k T)) I_{i k}(x(k T), x((k-1) T))\right]^{T} \\
& \times P[x(k T) \\
& \left.\quad+\sum_{i=1}^{r} h_{i}(z(k T)) I_{i k}(x(k T), x((k-1) T))\right] \\
& \leq \lambda_{2}[x(k T) \\
& \left.\quad+\sum_{i=1}^{r} h_{i}(z(k T)) I_{i k}(x(k T), x((k-1) T))\right]^{T} \\
& \times \sum_{i=1}^{r} h_{i}(z(k T)) \\
& \quad \times\left[x(k T)+\sum_{i k}(x(k T), x((k-1) T))\right] \\
& \quad x(k T) \\
& \quad+\sum_{i=1}^{r} h_{i}(z(k T)) h_{j}(z(k T)) \\
& \quad h_{i=1}^{r}(z(k T)) \\
& \times\left[x(k T)+I_{i k}(x(k T), x((k-1) T)) I_{i k}(x(k T), x((k-1) T))\right]
\end{aligned}
$$

$$
\begin{aligned}
& \leq \lambda_{2} \sum_{i=1}^{r} \sum_{j=1}^{r} h_{i}(z(k T)) h_{j}(z(k T)) \\
& \times\left[\frac{\theta}{2}\left\|x(k T)+I_{i k}(x(k T), x((k-1) T))\right\|^{2}\right. \\
& \left.\quad+\frac{1}{2 \theta}\left\|x(k T)+I_{j k}(x(k T), x((k-1) T))\right\|^{2}\right] \\
& =\frac{\theta \lambda_{2}}{2} \sum_{i=1}^{r} h_{i}(z(k T)) \\
& \quad \times\left\|x(k T)+I_{i k}(x(k T), x((k-1) T))\right\|^{2} \\
& +\frac{\lambda_{2}}{2 \theta} \sum_{j=1}^{r} h_{j}(z(k T)) \\
& \quad \times\left\|x(k T)+I_{j k}(x(k T), x((k-1) T))\right\|^{2} \\
& \leq \lambda_{2}\left(\frac{\theta}{2}+\frac{1}{2 \theta}\right) \sum_{i=1}^{r} h_{i}(z(k T)) \\
& \quad \times\left[a_{i k}\|x(k T)\|^{2}+b_{i k}\|x((k-1) T)\|^{2}\right] \\
& +\frac{\left(\theta^{2}+1\right) \lambda_{2}}{2 \theta} \sum_{i=1}^{r} h_{i}(z(k T)) a_{i k} \omega(x(k T)) \\
& +1) \lambda_{2} \sum_{i=1}^{r} h_{i}(z(k T)) b_{i k} \omega(x((k-1) T)) .
\end{aligned}
$$

Hence, we have

$$
\omega\left(k T^{+}\right) \leq H_{k} \omega(k T)+B_{k} \omega((k-1) T) .
$$

From (27), we have

$$
\omega(t) \leq \eta \omega\left((k-1) T^{+}\right) \exp [\beta(t-(k-1) T)] \frac{G(t)}{G\left((k-1) T^{+}\right)}
$$

for $t \in((k-1) T, k T]$ and $k \in Z^{+}$.

Then for $t \in(0, T]$, we get

$$
\omega(t) \leq \omega(0) \eta \exp (\beta t) \frac{G(t)}{G\left(0^{+}\right)}
$$

which implies that

$$
\omega(T) \leq \omega(0) \eta \exp (\beta T) \frac{G(T)}{G\left(0^{+}\right)} .
$$


This together with (29) implies that

$$
\begin{aligned}
\omega\left(T^{+}\right) & \\
\leq & H_{1} \omega(T)+B_{1} \omega(0) \\
\leq & H_{1} \omega(0) \eta \exp (\beta T) \frac{G(T)}{G\left(0^{+}\right)}+B_{1} \omega(0) \\
= & {\left[H_{1}+\frac{B_{1}}{\eta} \exp (-\beta T) \frac{G\left(0^{+}\right)}{G(T)}\right] } \\
& \times \omega(0) \eta \exp (\beta T) \frac{G(T)}{G\left(0^{+}\right)} \\
= & L_{1} \omega(0) \eta \exp (\beta T) \frac{G(T)}{G\left(0^{+}\right)} .
\end{aligned}
$$

Similarly, for $t \in(T, 2 T]$, we obtain

$$
\begin{aligned}
\omega(t) \leq & \omega\left(T^{+}\right) \eta \exp [\beta(t-T)] \frac{G(t)}{G\left(T^{+}\right)} \\
\leq & L_{1} \omega(0) \eta^{2} \exp (\beta t) \frac{G(T)}{G\left(0^{+}\right)} \times \frac{G(t)}{G\left(T^{+}\right)}, \\
\omega\left(2 T^{+}\right) \leq & H_{2} \omega(2 T)+B_{2} \omega(T) \\
\leq & H_{2} L_{1} \omega(0) \eta^{2} \exp (2 \beta T) \frac{G(T)}{G\left(0^{+}\right)} \\
& \times \frac{G(2 T)}{G\left(T^{+}\right)}+B_{2} \omega(0) \eta \exp (\beta T) \frac{G(T)}{G\left(0^{+}\right)} \\
= & L_{1}\left[H_{2}+\frac{B_{2}}{\eta L_{1}} \exp (-\beta T) \frac{G\left(T^{+}\right)}{G(2 T)}\right] \\
& \times \omega(0) \eta^{2} \exp (2 \beta T) \frac{G(T)}{G\left(0^{+}\right)} \times \frac{G(2 T)}{G\left(T^{+}\right)} \\
= & L_{1} \times L_{2} \omega(0) \eta^{2} \exp (2 \beta T) \frac{G(T)}{G\left(0^{+}\right)} \times \frac{G(2 T)}{G\left(T^{+}\right)} .
\end{aligned}
$$

Further for $t \in(2 T, 3 T]$, we have

$$
\begin{aligned}
\omega(t) \leq & \omega\left(2 T^{+}\right) \eta \exp [\beta(t-2 T)] \frac{G(t)}{G\left(2 T^{+}\right)} \\
\leq & L_{1} \times L_{2} \omega(0) \eta^{3} \exp (\beta t) \frac{G(T)}{G\left(0^{+}\right)} \\
& \times \frac{G(2 T)}{G\left(T^{+}\right)} \times \frac{G(t)}{G\left(2 T^{+}\right)} .
\end{aligned}
$$

Hence, generally, for $t \in((k-1) T, k T]$, we obtain

$$
\begin{aligned}
\omega(t) \leq & L_{0} \times L_{1} \times L_{2} \times \cdots \times L_{k-1} \omega(0) \eta^{k} \exp (\beta t) \\
& \times \frac{G(T)}{G\left(0^{+}\right)} \times \frac{G(2 T)}{G\left(T^{+}\right)} \times \cdots \times \frac{G(t)}{G\left((k-1) T^{+}\right)},
\end{aligned}
$$

where $L_{0}=1$ and $k \in Z^{+}$.
In the following, we consider the following two cases.

Case 1. $\beta \geq 0$; that is, condition (1) holds.

It follows from (21) that

$$
L_{k} \eta \exp (\beta T) \frac{G((k+1) T)}{G\left(k T^{+}\right)} \leq \frac{1}{\mu}, \quad \forall k \in Z^{+} .
$$

This combined with (36) yields

$$
\begin{aligned}
\omega(t) \leq & L_{1} \times L_{2} \times \cdots \times L_{k-1} \omega(0) \eta^{k} \exp (k \beta T) \\
& \times \frac{G(T)}{G\left(0^{+}\right)} \times \frac{G(2 T)}{G\left(T^{+}\right)} \times \cdots \times \frac{G(t)}{G\left((k-1) T^{+}\right)} \\
= & \omega(0) \eta \exp (\beta T) \frac{G(t) G(T)}{G\left(0^{+}\right) G(k T)} \\
& \times L_{1} \eta \exp (\beta T) \frac{G(2 T)}{G\left(T^{+}\right)} \\
& \times L_{2} \eta \exp (\beta T) \frac{G(3 T)}{G\left(2 T^{+}\right)} \\
& \times \cdots \times L_{k-1} \eta \exp (\beta T) \frac{G(k T)}{G\left((k-1) T^{+}\right)} \\
\leq & \frac{\eta M^{2}}{m^{2} \mu^{k-1}} \omega(0) \exp (\beta T) .
\end{aligned}
$$

Note that $k \rightarrow \infty$ as $t \rightarrow \infty$; from $\mu>1$, we obtain $\lim _{t \rightarrow \infty} \omega(t)=0$.

Case 2. $\beta<0$; that is, condition (2) holds.

It follows from (22) that

$$
\eta L_{k} \exp (-\varepsilon T) \frac{G((k+1) T)}{G\left(k T^{+}\right)} \leq 1, \quad \forall k \in Z^{+} .
$$

So, we have

$$
\begin{aligned}
\omega(t) \leq & L_{1} \times L_{2} \times \cdots \times L_{k-1} \omega(0) \eta^{k} \exp (-\varepsilon t) \\
& \times \exp [(\beta+\varepsilon) t] \\
& \times \frac{G(T)}{G\left(0^{+}\right)} \times \frac{G(2 T)}{G\left(T^{+}\right)} \times \cdots \times \frac{G(t)}{G\left((k-1) T^{+}\right)} \\
\leq & \omega(0) \eta \exp [(\beta+\varepsilon) t] \frac{G(t) G(T)}{G\left(0^{+}\right) G(k T)} \\
& \times L_{1} \eta \exp (-\varepsilon T) \frac{G(2 T)}{G\left(T^{+}\right)} \\
& \times L_{2} \eta \exp (-\varepsilon T) \frac{G(3 T)}{G\left(2 T^{+}\right)} \\
& \times \cdots \times L_{k-1} \eta \exp (-\varepsilon T) \frac{G(k T)}{G\left((k-1) T^{+}\right)} \\
\leq & \frac{M^{2}}{m^{2}} \omega(0) \eta \exp [(\beta+\varepsilon) t] .
\end{aligned}
$$

Hence, from $\varepsilon+\beta<0$, we obtain $\lim _{t \rightarrow \infty} \omega(t)=0$. 
From the above discussion, we know that the trivial solution $x(t) \equiv 0$ of system (16) is asymptotically stable. This completes the proof of Theorem 6 .

Remark 7. If $\mu=1$ or $\varepsilon=-\beta$ in Theorem 6 , from the proved process of Theorem 6 , we have

$$
\omega(t) \leq \frac{\eta M^{2} Q}{m^{2}} \omega(0),
$$

where $Q=\max \{1, \exp (\beta T)\}$. In this case, it is clear that the origin of system (16) is stable. In addition, under condition (2) in Theorem 6, it is evident that the origin of system (16) is globally exponentially stable.

Remark 8. If $H_{k} \equiv H$ for all $k \in Z^{+}$in (18), inequality (21) can be replaced by the following inequality:

$$
\beta T+\ln \left(\mu \eta \Lambda_{k}\right)+\ln \frac{G((k+1) T)}{G\left(k T^{+}\right)} \leq 0 \quad \forall k \in Z^{+},
$$

where $H$ is a constant and $\Lambda_{k}=H+$ $\left(B_{k} / \eta H\right) \exp (-\beta T)\left(G\left((k-1) T^{+}\right) / G(k T)\right)$. In fact, from the definition of $L_{k}$, we have $L_{k} \geq H$ for all $k \in Z^{+}$in this case, which implies that $L_{k} \leq \Lambda_{k}$ for all $k \in Z^{+}$. Hence, from (36), we obtain

$$
\begin{aligned}
\omega(t) \leq & \Lambda_{0} \times \Lambda_{1} \times \Lambda_{2} \times \cdots \times \Lambda_{k-1} \eta^{k} \omega(0) \exp (\beta t) \\
& \times \frac{G(T)}{G\left(0^{+}\right)} \times \frac{G(2 T)}{G\left(T^{+}\right)} \times \cdots \times \frac{G(t)}{G\left((k-1) T^{+}\right)},
\end{aligned}
$$

for $t \in((k-1) T, k T]$, where $\Lambda_{0}=1$. Similar to the analytic technique of (36), we know that $\lim _{t \rightarrow \infty} \omega(t)=0$ under condition $\left(21^{\prime}\right)$.

Similarly, in this case, inequality (22) can be replaced by the following inequality:

$$
\ln \left(\eta \Lambda_{k}\right)-\varepsilon T+\ln \frac{G((k+1) T)}{G\left(k T^{+}\right)} \leq 0, \quad \forall k \in Z^{+} .
$$

Let $G(t)$ be a constant function and $P=I$ in Theorem 6; then $\eta=1$ and $\beta=\alpha=\max _{1 \leq i \leq r}\left\{\beta_{i}\right\}$, where $I$ is the identity matrix and $\beta_{i}$ denotes the largest eigenvalue of $\left(A_{i}+F_{i}\right)+$ $\left(A_{i}+F_{i}\right)^{T}$. Besides, let $I_{i k}(x(t), x(t-T))=I_{k}(x(t), x(t-T))$ for $i=1,2, \ldots, r$ and $k \in Z^{+}$. In this case, $a_{i k}$ and $b_{i k}$ are reduced to $a_{k}$ and $b_{k}$, respectively. $H_{k}, B_{k}$, and $L_{k}$ can be rewritten as

$$
\begin{aligned}
& \bar{H}_{k}=\frac{\left(\theta^{2}+1\right)}{2 \theta} a_{k}, \quad k \in Z^{+}, \\
& \bar{B}_{k}=\frac{\left(\theta^{2}+1\right)}{2 \theta} b_{k}, \quad k \in Z^{+}, \\
& \bar{L}_{k}=\bar{H}_{k}+\frac{\bar{B}_{k}}{\bar{L}_{k-1}} \exp (-\beta T), \quad k \in Z^{+},
\end{aligned}
$$

where $\bar{L}_{0}=1$. Under those assumptions, the following results are directly obtained from Theorem 6.
Corollary 9. Under assumption (H), system (16) is asymptotically stable at its origin, if one of the following conditions is satisfied.

(1) $\beta \geq 0$ and there exists a constant $\mu>1$ such that

$$
\beta T+\ln \left(\mu \bar{L}_{k}\right) \leq 0 \quad \forall k \in Z^{+} .
$$

(2) $\beta<0$ and there exists a constant $\varepsilon$ satisfying $0 \leq \varepsilon<$ $-\beta$ such that

$$
\ln \bar{L}_{k}-\varepsilon T \leq 0 \quad \forall k \in Z^{+} .
$$

In the following, we consider the case where the impulsive controller is reduced to linearly impulsive controller; that is,

$$
I_{i k}(x(t), x(t-T))=K_{i k} x(t)+C_{i k} x(t-T)
$$

for $i=1,2, \ldots, r$ and $k \in Z^{+}$in Theorem 6 , where each $K_{i k}$ and $C_{i k}$ are the $n \times n$ constant matrices; then system (16) can be rewritten as the following form:

$$
\begin{array}{r}
\dot{x}(t)=\sum_{i=1}^{r} h_{i}(z(t))\left(A_{i}+F_{i}\right) x(t), \quad t \neq k T, \\
\Delta x(t)=\sum_{i=1}^{r} h_{i}(z(t))\left[K_{i k} x(t)+C_{i k} x(t-T)\right], \\
t=k T, k \in Z^{+} .
\end{array}
$$

In this case, we obtain

$$
\begin{aligned}
\| u+ & I_{i k}(u, v) \|^{2} \\
= & \left(u+K_{i k} u+C_{i k} v\right)^{T}\left(u+K_{i k} u+C_{i k} v\right) \\
= & u^{T}\left(I+K_{i k}\right)^{T}\left(I+K_{i k}\right) u \\
& +2 u^{T}\left(I+K_{i k}\right)^{T} C_{i k} v+v^{T} C_{i k}^{T} C_{i k} v \\
\leq & 2 u^{T}\left(I+K_{i k}\right)^{T}\left(I+K_{i k}\right) u+2 v^{T} C_{i k}^{T} C_{i k} v \\
\leq & \gamma_{i k} u^{T} u+\rho_{i k} v^{T} v,
\end{aligned}
$$

where $\gamma_{i k} \geq 2 \lambda_{\max }\left[\left(I+K_{i k}\right)^{T}\left(I+K_{i k}\right)\right]$ and $\rho_{i k} \geq$ $2 \lambda_{\max }\left(C_{i k}^{T} C_{i k}\right) ; I$ is the identity matrix.

Let $P=I$ and

$$
\begin{array}{ll}
\mathbb{H}_{k}=\sum_{i=1}^{r} h_{i}(z(k T)) \gamma_{i k}, & k \in Z^{+}, \\
\mathbb{B}_{k}=\sum_{i=1}^{r} h_{i}(z(k T)) \rho_{i k}, \quad k \in Z^{+} .
\end{array}
$$

In addition, let $G(t)$ be a constant function and $\Delta_{k}=$ $\mathbb{H}_{k}+\left(\mathbb{B}_{k} / \Delta_{k-1}\right) \exp (-\beta T)$ for $k \in Z^{+}$, where $\Delta_{0}=1$. From Theorem 6 , we have the following results.

Corollary 10. System (47) is asymptotically stable at its origin, if one of the following conditions is satisfied. 
(1) $\beta \geq 0$ and there exists a constant $\mu>1$ such that

$$
\beta T+\ln \left(\mu \Delta_{k}\right) \leq 0, \quad \forall k \in Z^{+} .
$$

(2) $\beta<0$ and there exists a constant $\varepsilon$ satisfying $0 \leq \varepsilon<$ $-\beta$ such that

$$
\ln \Delta_{k}-\varepsilon T \leq 0, \quad \forall k \in Z^{+}
$$

Choose $K_{i k}=K$ and $C_{i k}=C$ for all $i=1,2, \ldots, r$ and $k \in Z^{+}$in system (47), where $K$ and $C$ are the $n \times n$ constant matrices, respectively. Let $\gamma \geq 2 \lambda_{\max }\left[(I+K)^{T}(I+K)\right]>0$ and $\rho \geq 2 \lambda_{\text {max }}\left(C^{T} C\right)$. Let $G(t)$ be a constant and $P=I$; then $\beta=\alpha=\max _{1 \leq i \leq r}\left\{\beta_{i}\right\}$, where $\beta_{i}$ denotes the largest eigenvalue of $\left(A_{i}+F_{i}\right)+\left(A_{i}+F_{i}\right)^{T}$. In addition, under those assumptions, $\eta=1, \mathbb{T}_{k}=\gamma$, and $\mathbb{B}_{k}=\rho$ for all $k \in Z^{+}$. Denote $\Gamma=$ $\gamma+(\rho / \gamma) \exp (-\beta T)$. The following results are directly obtained from Theorem 6 and Remark 8.

Corollary 11. For system (47), the following statements hold.

(1) If $\beta \geq 0$ and there exists a constant $\mu>1$ such that

$$
\beta T+\ln (\mu \Gamma) \leq 0,
$$

then the origin of system (47) is asymptotically stable.

(2) If $\beta<0$ and there exists a constant $\varepsilon$ satisfying $0 \leq \varepsilon<$ $-\beta$ such that

$$
\ln \Gamma-\varepsilon T \leq 0,
$$

then the origin of system (47) is exponentially stable.

Remark 12. From Corollary 11, we can obtain an estimate of the upper bound of $T$ when $\beta>0$. In fact, it follows from Corollary 11 that

$$
T \leq \frac{1}{\beta}\left[\ln (\gamma-\mu \rho)-\ln \left(\mu \gamma^{2}\right)\right]
$$

\section{Time-Delayed Fuzzy Impulsive Synchronization}

The synchronization of chaotic system based on T-S fuzzy model has been extensively studied in previous researches. For instance, $[13,15,16]$ obtained some results on the synchronization of chaotic system by using fuzzy impulsive control technique and adaptive synchronization approach, respectively. But, to the best of our knowledge, there are few results on the synchronization of chaotic system based on T-S fuzzy model by applying time-delayed impulsive control method. In this section, we consider time-delayed impulsive synchronization of chaotic systems based on T-S fuzzy model. In order to deal with synchronization of chaotic systems, we need to design the control input so that the driven system achieves asymptotic synchronization with the driving system, provided that the two systems start from different initial conditions. The driving system is given by system (7), suppose that the driven system has the same premise variables as the driving system, and the output states of driving system are observable. The fuzzy rules of the driven system are presented by the following form.

Rule $i$ : if $z_{1}(t)$ is $M_{i}^{1}, z_{2}(t)$ is $M_{i}^{2}, \ldots, z_{p}(t)$ is $M_{i}^{p}$, then

$$
\dot{y}(t)=A_{i} y(t)+u_{i}(t)
$$

where $z_{j}(t), M_{i}^{j}$, and $A_{i}$ are defined in system (2), and $y(t)$ is the state variable of the driven system; let $u_{i}(t)$ be the external control inputs represented by the following form:

$$
\begin{aligned}
u_{i}(t)=\sum_{k=1}^{\infty} F_{i} l_{k}(t)[y(t)-x(t)] \\
+\sum_{k=1}^{\infty} \delta(t-k T) \\
\quad \quad \times I_{i k}(y(t)-x(t), y(t-T)-x(t-T)),
\end{aligned}
$$

where $x(t)$ is the state variable of the driving system, $I_{i k}(\cdot)$ is defined in (10) satisfying assumption $(H)$, and $I_{i k}(0,0)=0$.

Using the same methods in Section 3, the final output of the driven system is represented as follows:

$$
\begin{array}{r}
\dot{y}(t)=\sum_{i=1}^{r} h_{i}(z(t)) A_{i} y(t)+\sum_{i=1}^{r} h_{i}(z(t)) F_{i}[y(t)-x(t)], \\
t \neq k T, \\
\Delta y(t)=\sum_{i=1}^{r} h_{i}(z(t)) I_{i k}(y(t)-x(t), y(t-T)-x(t-T)), \\
t=k T,
\end{array}
$$

where $k \in Z^{+}$

Let $e(t)=y(t)-x(t)$; then from (7) and (57), the error system can be expressed as the following form:

$$
\begin{array}{r}
\dot{e}(t)=\sum_{i=1}^{r} h_{i}(z(t))\left(A_{i}+F_{i}\right) e(t), \quad t \neq k T, \\
\Delta e(t)=\sum_{i=1}^{r} h_{i}(z(t)) I_{i k}(e(t), e(t-T)), \\
t=k T, k \in Z^{+} .
\end{array}
$$

We observe that the synchronization of driving system (7) and driven system (57) is converted to the asymptotical stability of the origin for system (58). On the other hand, it is evident that error system (58) is similar to system (16), and then similar to Theorem 6, we have the following statements, which guarantee the synchronization of drivingdriven systems (7) and (57).

Theorem 13. For driving-driven systems (7) and (57), the following statements are true. 
(1) Systems (7) and (57) are globally asymptotically synchronized, if $\beta \geq 0$ and there exists a constant $\mu>1$ such that

$$
\beta T+\ln \left(\mu \eta L_{k}\right)+\ln \frac{G((k+1) T)}{G\left(k T^{+}\right)} \leq 0, \quad \forall k \in Z^{+} .
$$

(2) Systems (7) and (57) are globally exponentially synchronized, if $\beta<0$ and there exists a constant $\varepsilon$ satisfying $0 \leq \varepsilon<-\beta$ such that

$$
\ln \left(\eta L_{k}\right)-\varepsilon T+\ln \frac{G((k+1) T)}{G\left(k T^{+}\right)} \leq 0 \quad \forall k \in Z^{+} .
$$

Theorem 13 can be proved by using a similar argument as the proof of Theorem 6, and we omit it here.

Remark 14. If the impulsive controllers $I_{i k}$ reduce to the linear impulsive forms (46), similar to the above discussion in Section 3, it is easy to see that driving-driven systems (7) and (57) are globally asymptotically synchronized if the conditions of Corollaries 10 and 11 are satisfied, respectively.

Remark 15. Evidently, Theorems 6 and 13 are established based on the fact that the impulsive internals are equidistant and equivalent to the feedback time-delay $T$. For the generally impulsive sequences $\left\{t_{k}\right\}_{k=1}^{\infty}$ satisfying $0<t_{1}<t_{2}<\cdots<t_{k}<$ $t_{k+1} \cdots$ and $\lim _{k \rightarrow \infty} t_{k}=\infty$, the corresponding results are not given because of the complexity of this case in this paper. Hence, an important and interesting problem is whether we can also obtain the similar results to guarantee the stability and synchronization of the addressed chaotic system based on the generally impulsive sequences.

\section{Numerical Simulations}

In this section, based on the results obtained in the previous sections, some numerical simulations are presented to show the effectiveness and feasibility of our results. form:

Consider the Rössler system described by the following

$$
\begin{gathered}
\dot{x}_{1}=-10 x_{1}+10 x_{2}, \\
\dot{x}_{2}=28 x_{1}-x_{2}-x_{1} x_{3}, \\
\dot{x}_{3}=x_{1} x_{2}-\frac{8}{3} x_{3} .
\end{gathered}
$$

Figure 1 shows that system (61) is chaotic. In the following, firstly, we consider time-delayed impulsive control of system (61) based on T-S fuzzy model.

Now, we can construct an exact T-S fuzzy model of system (61), which can be expressed as follows:

Rule 1: if $x_{1}$ is about $M_{1}$, then $\dot{x}=A_{1} x(t)+u_{1}(t)$,

Rule 2: if $x_{1}$ is about $M_{2}$, then $\dot{x}=A_{2} x(t)+u_{2}(t)$,

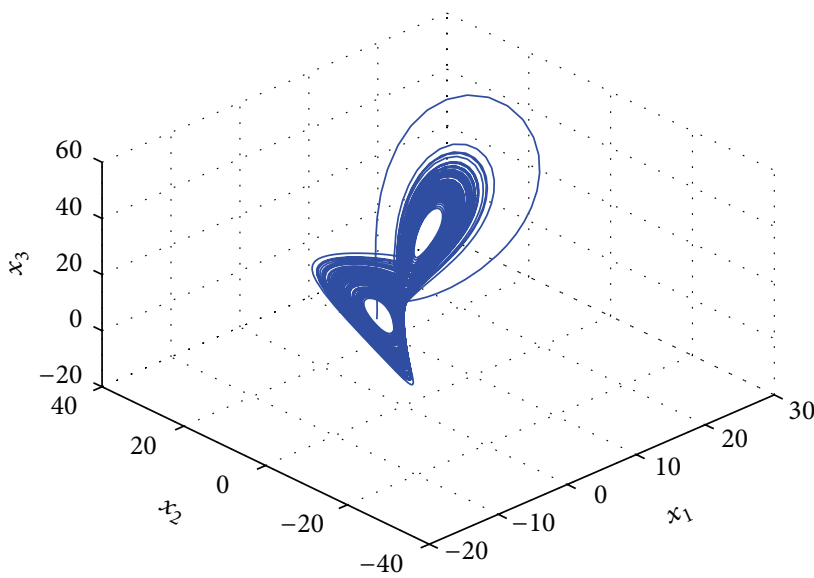

FIGURE 1: The chaotic behavior of system (61).
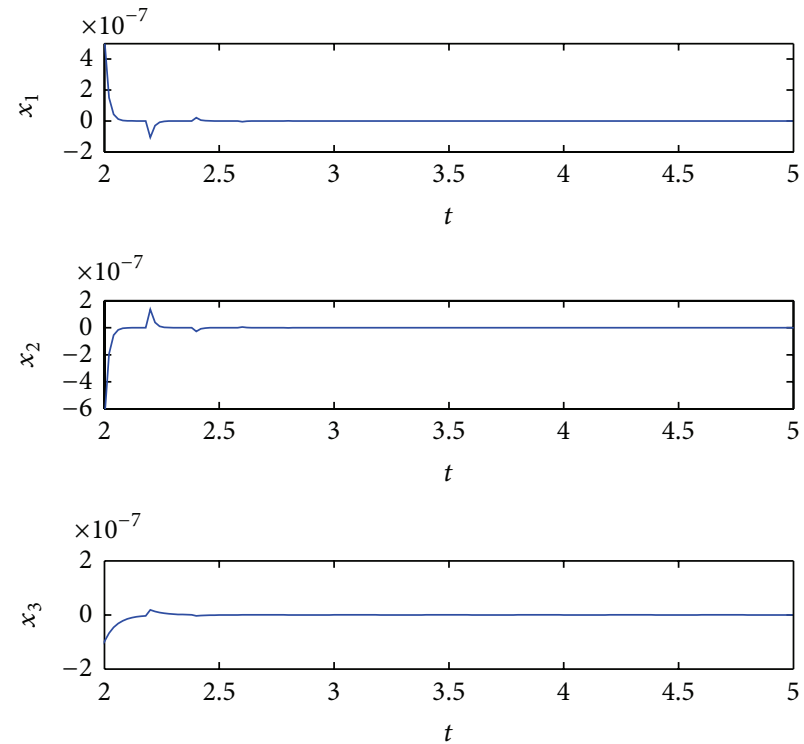

Figure 2: Time response of system (61).
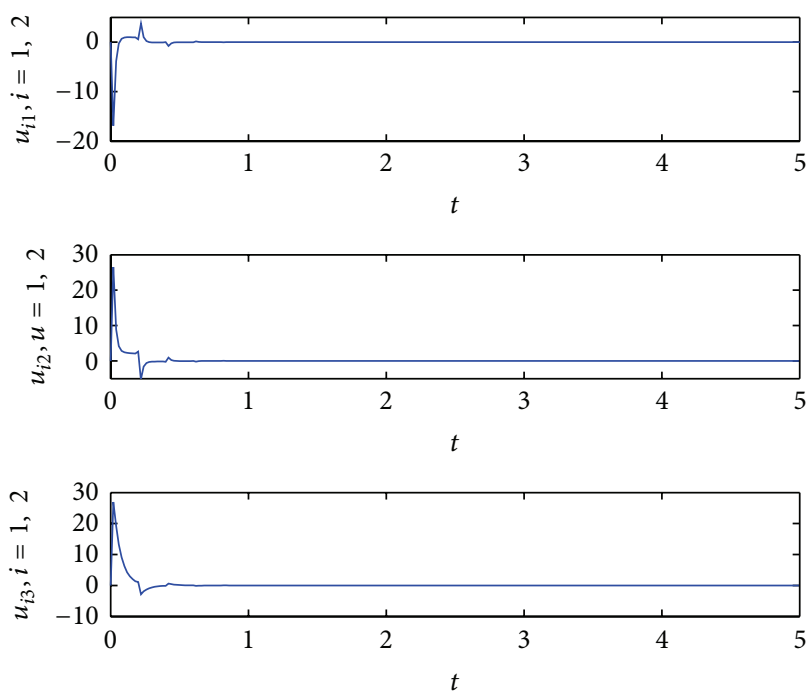

FIGURE 3: The plots of the control inputs. 

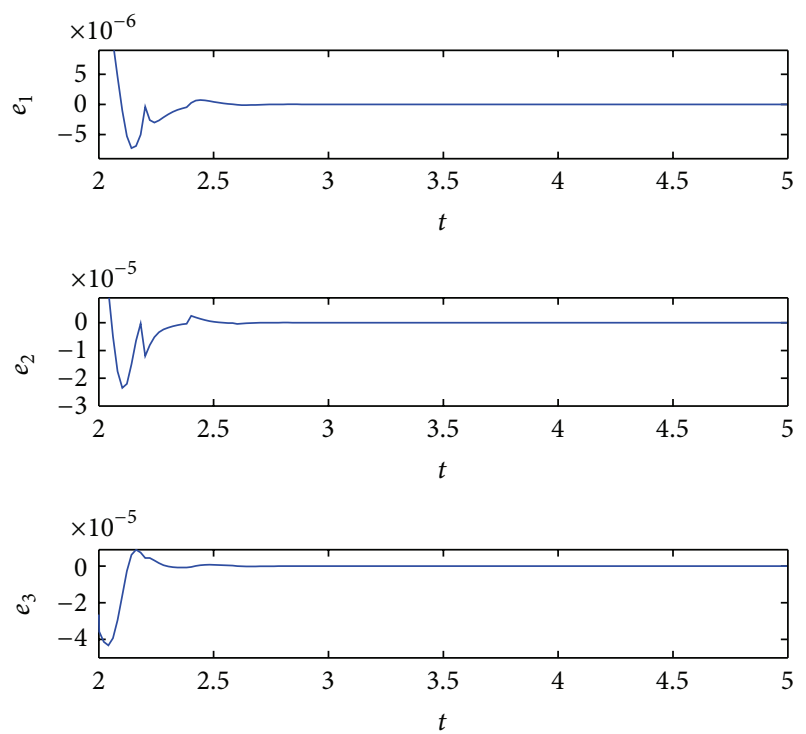

FIgURE 4: The plot of the synchronization errors.

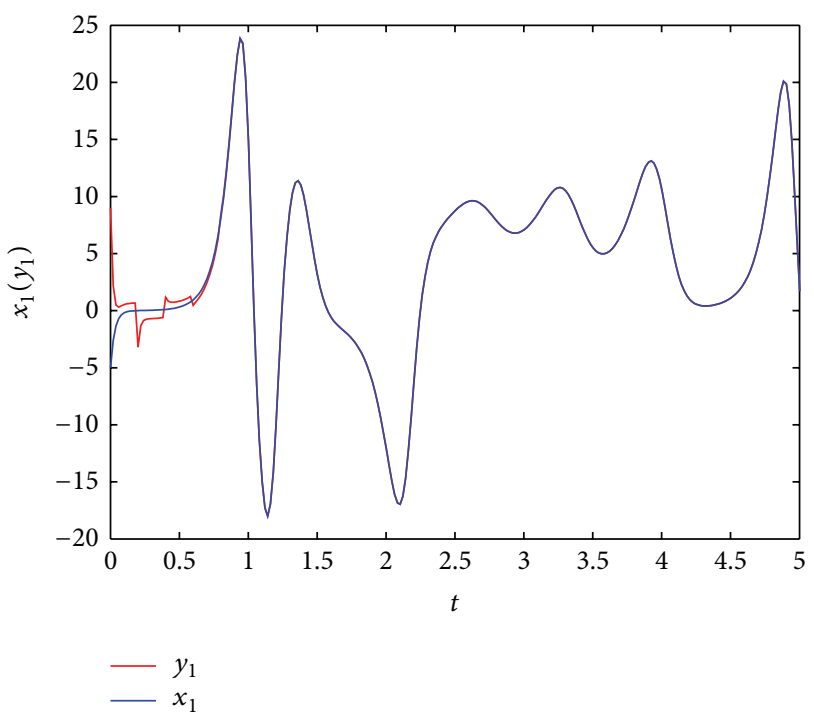

Figure 5: The curve of $x_{1}$ and $y_{1}$.

where $x(t)=\left(x_{1}(t), x_{2}(t), x_{3}(t)\right)^{T}, M_{1}=-30, M_{2}=30$,

$$
\begin{aligned}
& A_{1}=\left(\begin{array}{ccc}
-10 & 10 & 0 \\
28 & -1 & -30 \\
0 & 30 & -\frac{8}{3}
\end{array}\right), \\
& A_{2}=\left(\begin{array}{ccc}
-10 & 10 & 0 \\
28 & -1 & 30 \\
0 & -30 & -\frac{8}{3}
\end{array}\right),
\end{aligned}
$$

and the membership functions are $M_{1}\left(x_{1}\right)=(1 / 2)-\left(x_{1} / 2 d\right)$ and $M_{2}\left(x_{1}\right)=(1 / 2)+\left(x_{1} / 2 d\right)$ with $d=30$.

From (10), the delayed impulsive controllers can be designed in the following form.

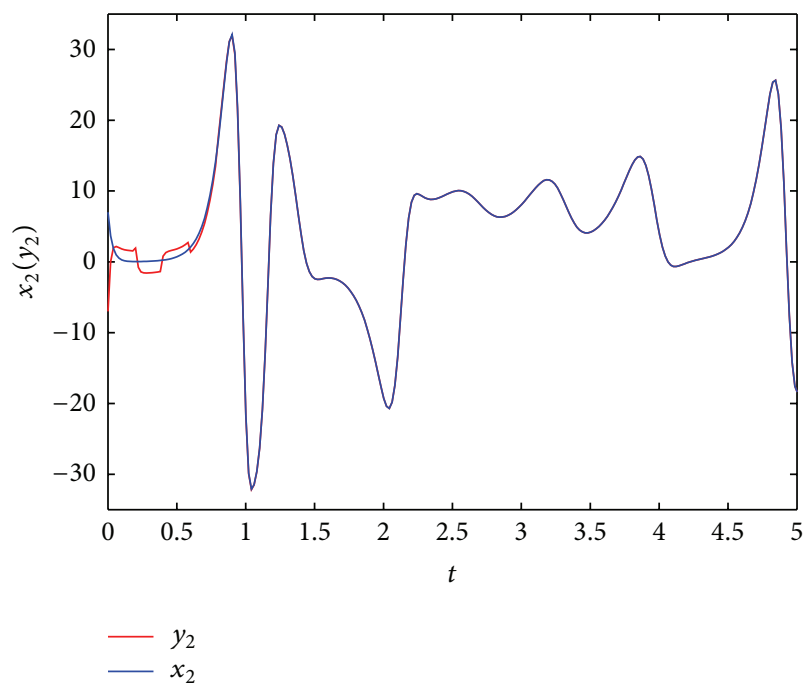

Figure 6: The curve of $x_{2}$ and $y_{2}$.

Control Rule 1: if $x_{1}$ is about $M_{1}$, then

$$
\begin{aligned}
u_{1}(t)= & \sum_{k=1}^{\infty} F_{1} l_{k}(t) x(t) \\
& +\sum_{k=1}^{\infty} \delta(t-k T)\left[K_{1} x(t)+C_{1} x(t-T)\right] .
\end{aligned}
$$

Control Rule 2: if $x_{1}$ is about $M_{2}$, then

$$
\begin{aligned}
u_{2}(t)= & \sum_{k=1}^{\infty} F_{2} l_{k}(t) x(t) \\
& +\sum_{k=1}^{\infty} \delta(t-k T)\left[K_{2} x(t)+C_{2} x(t-T)\right] .
\end{aligned}
$$

From system (16), the overall time-delayed impulsive control system of system (61) based on T-S fuzzy model can be rewritten as

$$
\begin{array}{r}
\dot{x}(t)=\sum_{i=1}^{2} M_{i}\left(x_{1}(t)\right)\left(A_{i}+F_{i}\right) x(t), \quad t \neq k T, \\
\Delta x(t)=\sum_{i=1}^{2} M_{i}\left(x_{1}(t)\right)\left[K_{i} x(t)+C_{i} x(t-T)\right], \\
t=k T, k \in Z^{+} .
\end{array}
$$

Choose $F_{1}=F_{2}=\operatorname{diag}(-13,-13,-13), K_{1}=K_{2}=$ $\operatorname{diag}(-0.6,-0.6,-0.6)$, and $C_{1}=C_{2}=\operatorname{diag}(-0.2,-0.2,-0.2)$; let $G(t)$ be a constant function and $P=I$. By simple computation, we obtain $\eta=1, \beta=2.0512, \gamma=0.32$, and $\rho=0.08$. Let $\mu=1.3$; then from Remark 12, we have $0 \leq T \leq$ 0.2360 . Here, we choose $T=0.2$. Hence, from Corollary 11 , we know that the origin of system (61) is asymptotically stable. The simulation results of stabilizing chaotic system (61) for $T=0.2$ are shown in Figure 2. Besides, it is evident that $u_{1}(t)$ 
and $u_{2}(t)$ are equivalent under the above parameters, which are simulated in Figure 3.

In the second simulation, we study time-delayed impulsive synchronization of the chaotic system (61) based on T-S fuzzy model in Figure 4.

The driving system is given in (62) with $u_{1}(t)=u_{2}(t)=$ 0 . Assume that the states of the delayed impulsive controlled driven system are $y(t)=\left(y_{1}(t), y_{2}(t), y_{3}(t)\right)^{T}$, and the fuzzy rules of the driven system are represented by

Rule 1 : if $x_{1}$ is about $M_{1}$, then $\dot{y}=A_{1} y(t)+u_{1}(t)$,

Rule 2: if $x_{1}$ is about $M_{2}$, then $\dot{y}=A_{2} y(t)+u_{2}(t)$,

$$
\begin{aligned}
u_{1}(t)= & \sum_{k=1}^{\infty} F_{1} l_{k}(t) e(t) \\
& +\sum_{k=1}^{\infty} \delta(t-k T)\left[K_{1} e(t)+C_{1} e(t-T)\right], \\
u_{2}(t)= & \sum_{k=1}^{\infty} F_{2} l_{k}(t) e(t) \\
& +\sum_{k=1}^{\infty} \delta(t-k T)\left[K_{2} e(t)+C_{2} e(t-T)\right],
\end{aligned}
$$

where $e(t)=y(t)-x(t), M_{i}$ and $A_{i}$ are defined in (62), $F_{i}$, $K_{i}$, and $C_{i}$ are chosen in (65), respectively, and the feedback delay $T=0.2$.

Then from (58), we know that the error system can be expressed as follows:

$$
\begin{array}{r}
\dot{e}(t)=\sum_{i=1}^{2} M_{i}\left(x_{1}(t)\right)\left(A_{i}+F_{i}\right) e(t), \quad t \neq k T, \\
\Delta e(t)=\sum_{i=1}^{2} M_{i}\left(x_{1}(t)\right)\left[K_{i} e(t)+C_{i} e(t-T)\right], \\
t=k T, k \in Z^{+} .
\end{array}
$$

Let $G(t)$ be a constant function and $P=I$; from Remark 14 and the above discussion for the stability of system (61), we know that the origin of the error system (69) is asymptotically stable; that is, system (62) without the external input and system (67) are globally asymptotically synchronized. Figure 3 shows the simulation result of the stability of the origin for error system (69). The synchronization of driving system (62) with respect to initial value $x(0)=$ $(-5,7,-4)^{T}$ and of driven system $(67)$ with initial value $y(0)=$ $(9,-7,10)^{T}$ is shown in Figures 5, 6, and 7. The control inputs are depicted in Figure 8.

\section{Conclusion}

In the paper, by using impulsive control method and the delayed feedback control mean, the stabilization and synchronization of chaotic system based on T-S fuzzy model

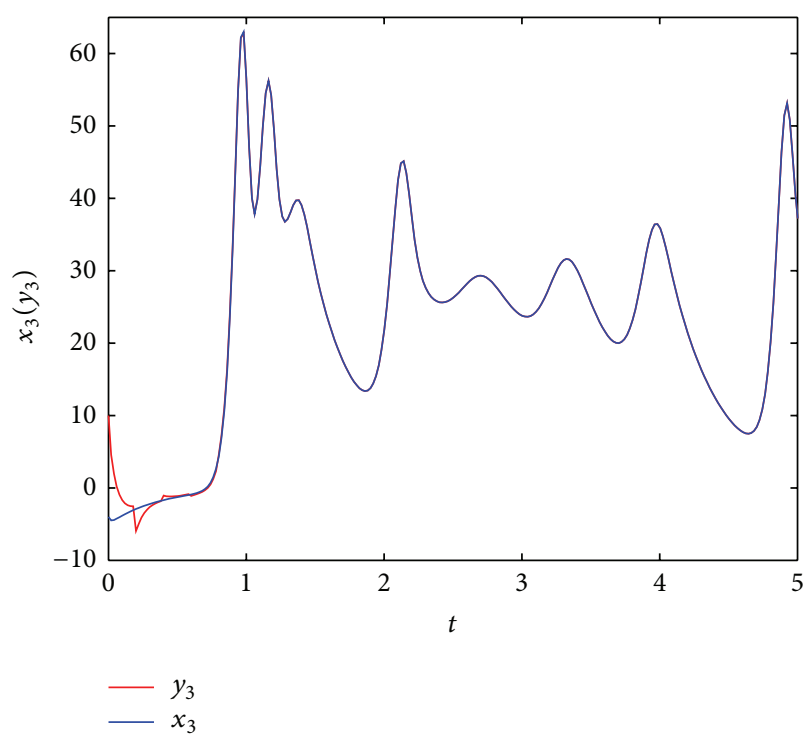

Figure 7: The curve of $x_{3}$ and $y_{3}$.
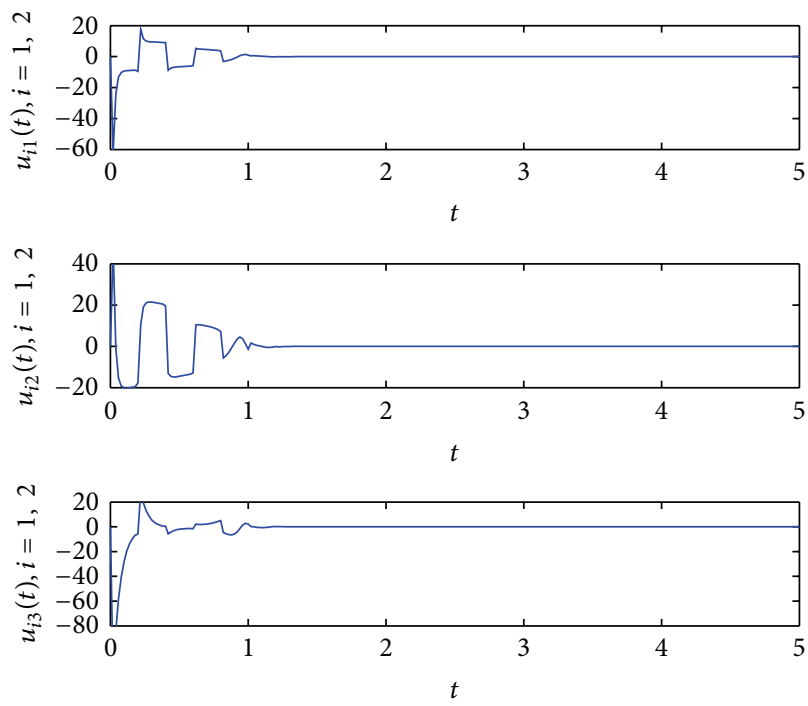

FIGURE 8: Time response of the control inputs.

are proposed; some useful and new conditions are obtained to ensure the stability and synchronization of the addressed chaotic system. In particular, some sufficient conditions which lead to the globally exponential stability of chaotic system are also obtained. It is noted that the impulsive functions can be nonlinear in this letter, which extend some previous results. Besides, in view of applying time-delayed feedback control technique, the delayed impulsive controllers are proposed in this paper. Finally, some numerical simulations are represented to show the effectiveness and feasibility of the developed methods.

\section{Conflict of Interests}

The authors declare that there is no conflict of interests regarding the publication of this paper. 


\section{Acknowledgment}

This work was supported by the Natural Science Foundation of Xinjiang (Grant no. 2013211B06).

\section{References}

[1] Z. Li, Fuzzy Chaotic Systems Modeling, Control and Applications, Springer, Berlin, Germany, 2006.

[2] Z. Li, W. A. Halang, and G. Chen, Integration of Fuzzy Logic and Chaos Theory, vol. 187 of Studies in Fuzziness and Soft Computing, Springer, Berlin, Germany, 2006.

[3] M. Danca, "Controlling chaos in discontinuous dynamical systems," Chaos, Solitons and Fractals, vol. 22, no. 3, pp. 605612, 2004.

[4] C. Grebogi and Y. C. Lai, "Controlling chaos in high dimensions," IEEE Transactions on Circuits and Systems. I. Fundamental Theory and Applications, vol. 44, no. 10, pp. 971-975, 1997.

[5] C. Grebogi, Y. Lai, and S. Hayes, "Control and applications of chaos," Journal of the Franklin Institute B, vol. 334, no. 5-6, pp. 1115-1146, 1997.

[6] C. Hua and X. Guan, "Adaptive control for chaotic systems," Chaos, Solitons \& Fractals, vol. 22, no. 1, pp. 55-60, 2004.

[7] E. Ott, C. Grebogi, and J. A. Yorke, "Controlling chaos," Physical Review Letters, vol. 64, no. 11, pp. 1196-1199, 1990.

[8] J. H. Park, "Controlling chaotic systems via nonlinear feedback control," Chaos, Solitons and Fractals, vol. 23, no. 3, pp. 10491054, 2005.

[9] K. Pyragas, "Control of chaos via an unstable delayed feedback controller," Physical Review Letters, vol. 86, no. 11, pp. 22652268, 2001.

[10] M. T. Yassen, "Chaos control of Chen chaotic dynamical system," Chaos, Solitons and Fractals, vol. 15, no. 2, pp. 271-283, 2003.

[11] J. Zhu and Y. P. Tian, "Stabilizing periodic solutions of nonlinear systems and applications in chaos control," IEEE Transactions on Circuits and Systems II: Express Briefs, vol. 52, no. 12, pp. 870874, 2005.

[12] J. Dong and G. H. Yang, "State feedback control of continuoustime T-S fuzzy systems via switched fuzzy controllers," Information Sciences, vol. 178, no. 6, pp. 1680-1695, 2008.

[13] C. Hu, H. Jiang, and Z. Teng, "Fuzzy impulsive control and synchronization of general chaotic system," Acta Applicandae Mathematicae, vol. 109, no. 2, pp. 463-485, 2010.

[14] L. H. Lan, "Stability analysis for a class of Takagi-Sugeno fuzzy control systems with PID controllers," International Journal of Approximate Reasoning, vol. 46, no. 1, pp. 109-119, 2007.

[15] J. H. Kim, C. W. Park, E. Kim, and M. Park, "Adaptive synchronization of T-S fuzzy chaotic systems with unknown parameters," Chaos, Solitons and Fractals, vol. 24, no. 5, pp. 13531361, 2005.

[16] X. Liu and S. Zhong, “T-S fuzzy model-based impulsive control of chaotic systems with exponential decay rate," Physics Letters A, vol. 370, no. 3-4, pp. 260-264, 2007.

[17] C.-S. Ting, "An observer-based approach to controlling timedelay chaotic systems via Takagi-Sugeno fuzzy model," Information Sciences, vol. 177, no. 20, pp. 4314-4328, 2007.

[18] Y. W. Wang, Z. H. Guan, and H. O. Wang, "Impulsive synchronization for Takagi-Sugeno fuzzy model and its application to continuous chaotic system," Physics Letters A, vol. 339, no. 3-5, pp. 325-332, 2005.
[19] X. Zhang, A. Khadra, D. Li, and D. Yang, "Impulsive stability of chaotic systems represented by T-S model," Chaos, Solitons and Fractals, vol. 41, no. 4, pp. 1863-1869, 2009.

[20] Y. Zheng and G. Chen, "Fuzzy impulsive control of chaotic systems based on TS fuzzy model," Chaos, Solitons and Fractals, vol. 39, no. 4, pp. 2002-2011, 2009.

[21] Q. Zhong, J. Bao, Y. Yu, and X. Liao, "Impulsive control for T-S fuzzy model-based chaotic systems," Mathematics and Computers in Simulation, vol. 79, no. 3, pp. 409-415, 2008.

[22] C. Chen, G. Feng, and X. Guan, "Robust synchronization of chaotic Luré systems via delayed feedback control," Physics Letters A, vol. 321, no. 5-6, pp. 344-354, 2004.

[23] Z. H. Guan, D. J. Hill, X. Shen, and X. Yu, "Synchronization of chaotic systems via hybrid impulsive and switching control," in Proceedings of the 5th Asian Control Conference, vol. 3, pp. 17621766, Melbourne, Australia, July 2004.

[24] P. Li and J. Cao, "Stabilisation and synchronisation of chaotic systems via hybrid control," IET Control Theory \& Applications, vol. 1, no. 3, pp. 795-801, 2007.

[25] C. Li, X. Liao, and R. Zhang, "A unified approach for impulsive lag synchronization of chaotic systems with time delay," Chaos, Solitons \& Fractals, vol. 23, no. 4, pp. 1177-1184, 2005.

[26] G. V. Osipov, A. K. Kozlov, and V. D. Shalfeev, "Impulse control of chaos in continuous systems," Physics Letters A, vol. 247, no. 1-2, pp. 119-128, 1998.

[27] T. Yang, L. B. Yang, and C. M. Yang, "Impulsive control of Lorenz system," Physica D: Nonlinear Phenomena, vol. 110, no. 1-2, pp. 18-24, 1997.

[28] G. Chen and X. Yu, "On time-delayed feedback control of chaotic systems," IEEE Transactions on Circuits and Systems I: Fundamental Theory and Applications, vol. 46, no. 6, pp. 767772, 1999.

[29] M. Chen, D. Zhou, and Y. Shang, "A simple time-delayed method to control chaotic systems," Chaos, Solitons and Fractals, vol. 22, no. 5, pp. 1117-1125, 2004.

[30] X. Guan, G. Feng, and C. Chen, "A stabilization method of chaotic systems based on full delayed feedback controller design," Physics Letters A, vol. 348, no. 3-6, pp. 210-221, 2006.

[31] J. Lu, Z. Ma, and L. Li, "Double delayed feedback control for the stabilization of unstable steady states in chaotic systems," Communications in Nonlinear Science and Numerical Simulation, vol. 14, no. 7, pp. 3037-3045, 2009.

[32] J. H. Park and O. M. Kwon, "A novel criterion for delayed feedback control of time-delay chaotic systems," Chaos, Solitons and Fractals, vol. 23, no. 2, pp. 495-501, 2005.

[33] N. Vasegh and A. K. Sedigh, "Chaos control in delayed chaotic systems via sliding mode based delayed feedback," Chaos, Solitons and Fractals, vol. 40, no. 1, pp. 159-165, 2009.

[34] M. de Sousa Vieira and A. J. Lichtenberg, "Controlling chaos using nonlinear feedback with delay," Physical Review E, vol. 54, no. 2, pp. 1200-1207, 1996. 


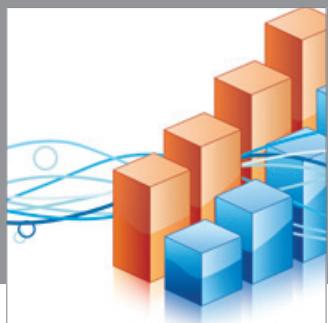

Advances in

Operations Research

mansans

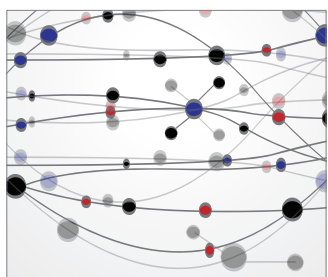

The Scientific World Journal
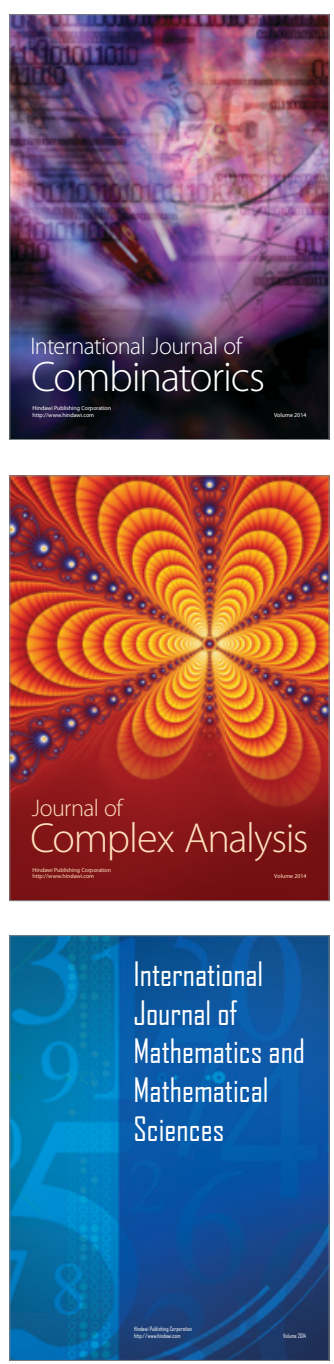
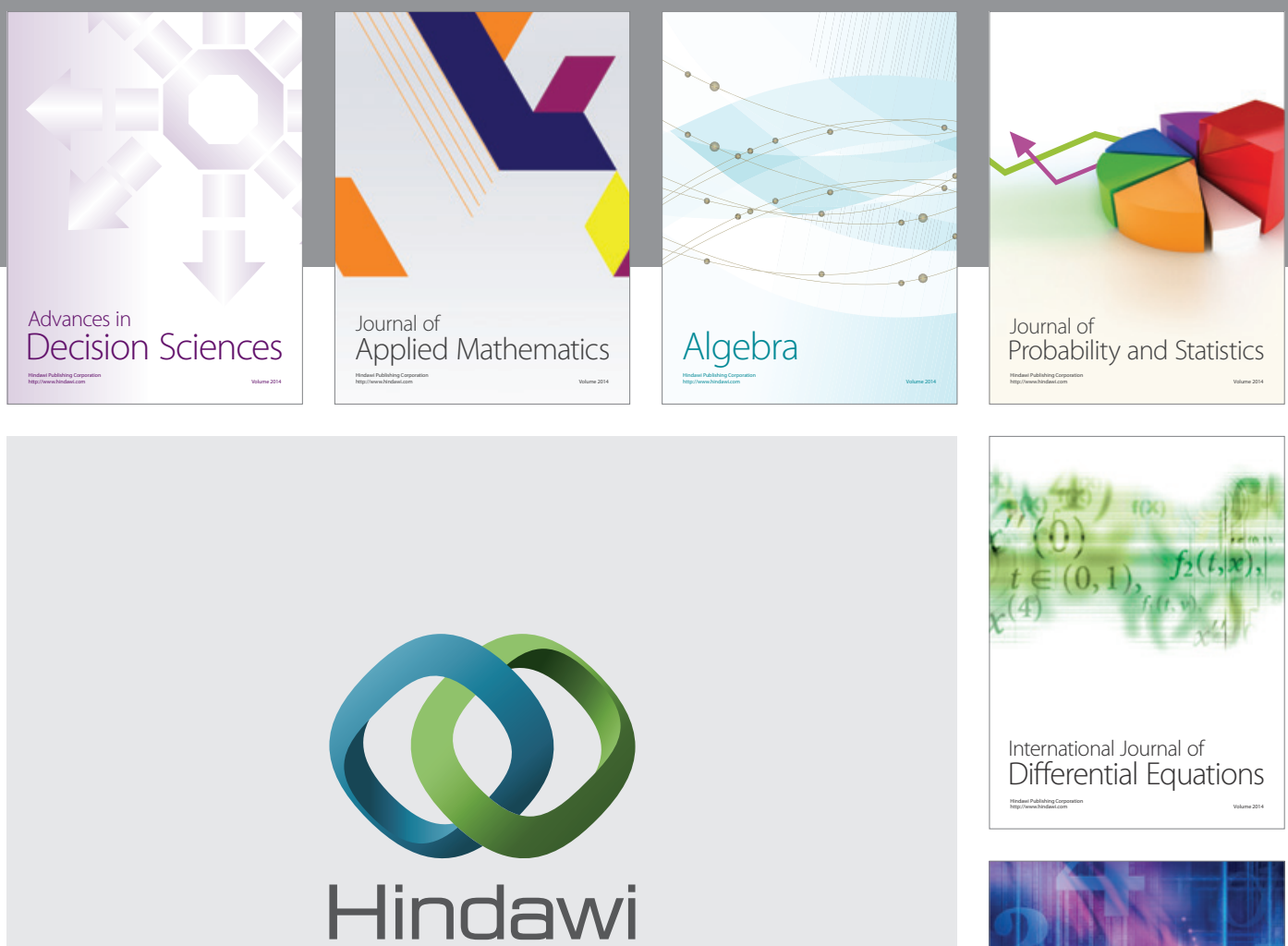

Submit your manuscripts at http://www.hindawi.com
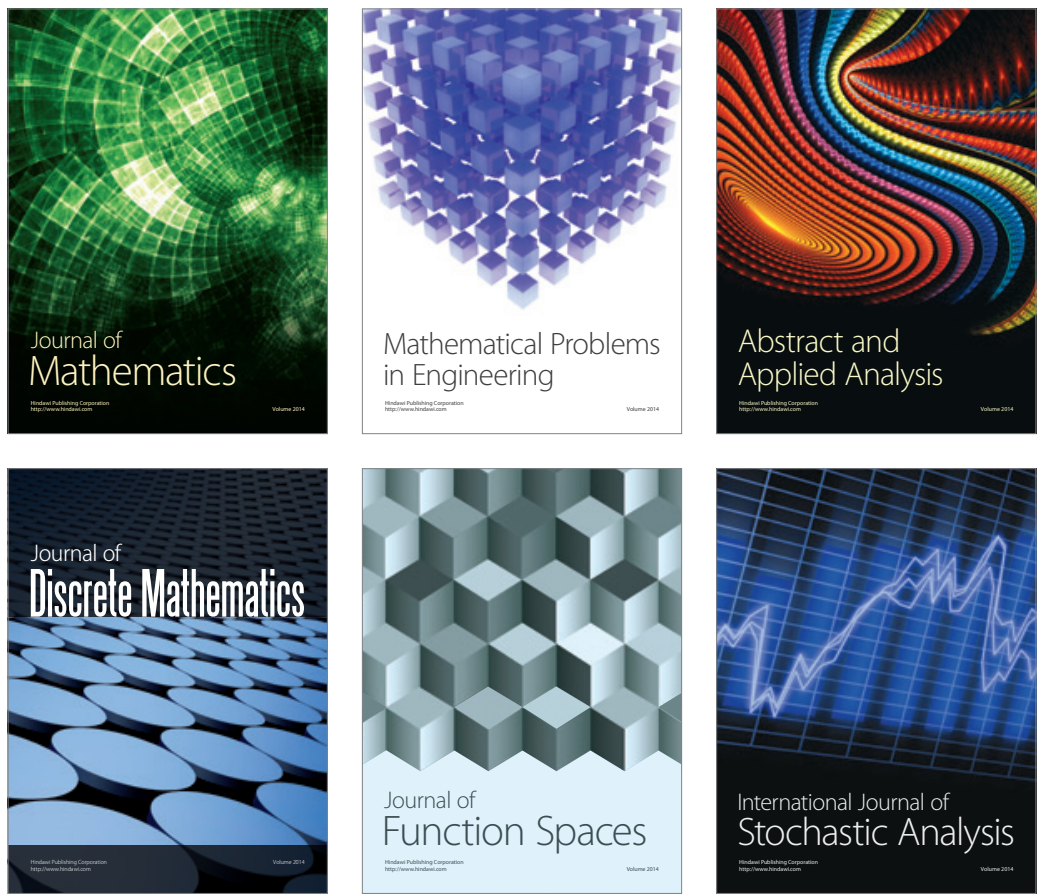

Journal of

Function Spaces

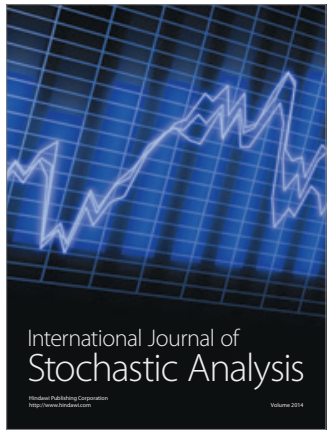

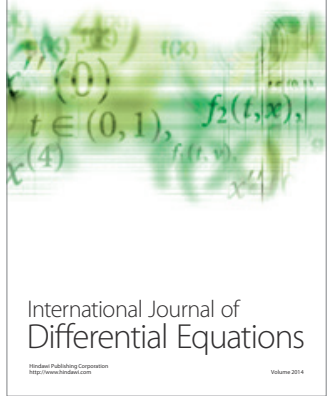
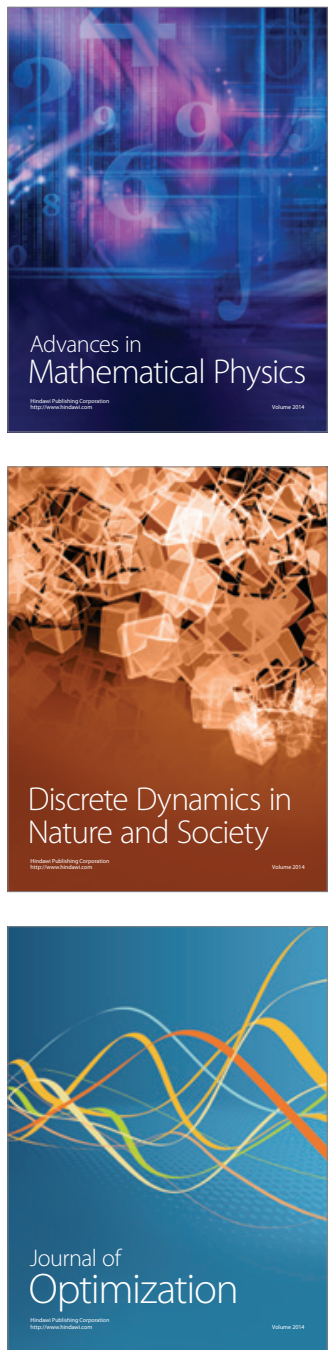